\title{
Prevalencia de síntomas de enfermedad de la superficie ocular en pacientes que acuden a consulta oftalmológica
}

\author{
Manuel Garza-León 1 , Everardo Hernández-Quintela², Héctor G. Cámara-Castillo³, \\ Paola de la Parra-Collin ${ }^{4}$, Paola Covarrubias-Espinosa ${ }^{5}$, Valeria Sánchez-Huerta², \\ Alejandro del Castillo-Ruiz', Fernando Rodríguez-Sixtos ${ }^{7}$, Jorge Pacheco-Patrón ${ }^{8}$, \\ Juan Carlos Ochoa-Tabares ${ }^{9}$, Karina Soto-Ortiz ${ }^{10}$ y Karen Hernández-Olguin ${ }^{11}$ en nombre del Grupo
} Nacional de Investigación en Ciencias Visuales

${ }^{1}$ Departamento de Ciencias Clínicas, División de Ciencias de la Salud, Universidad de Monterrey, Monterrey, N.L.; ${ }^{2}$ Servicio de Córnea y Cirugía Refractiva, Hospital Dr. Luis Sánchez Bulnes, Asociación para Evitar la Ceguera en México, I.A.P., Ciudad de México; ${ }^{3}$ Clínica de Mérida, Mérida, Yuc.; ${ }^{4}$ Clínica de Córnea y Superficie Ocular, Departamento de Oftalmología, Instituto Nacional de Rehabilitación, Ciudad de México; ${ }^{5}$ Hospital Aranda de la Parra, León, Gto.; ${ }^{6}$ Clínica Oftalmológica del Castillo S.C, Puebla, Pue.; ${ }^{7}$ Ocuclinic, Irapuato, Gto.; ${ }^{8}$ Hospital Ángeles, Chihuahua, Chih., y Hospital San José, Querétaro, Qro.; ${ }^{9}$ Servicios Optométricos y Ópticos SA de CV, Clínica Santa Lucía, Guadalajara, Jal.; ${ }^{10}$ Oftalmología Médica y Quirúrgica, Aguascalientes, Ags.; ${ }^{11}$ Instituto Mexicano del Seguro Social, Unidad Médica de Alta Especialidad No. 76, Hospital Mexicano-Americano, Nuevo Laredo, Tamps. México

\section{Resumen}

Objetivo: Determinar a través del cuestionario de enfermedad de la superficie ocular (OSDI, por sus siglas en inglés) la prevalencia de síntomas de la enfermedad de la superficie ocular y su relación con factores de riesgo asociados en pacientes que acuden a consulta oftalmológica. Método: Se realizó un estudio transversal entre septiembre y diciembre de 2014 para evaluar la prevalencia y los factores de riesgo de la enfermedad de la superficie ocular. Resultados: El valor promedio del OSDI fue de 40.46 × 23.62 puntos; el $86.4 \%$ de los pacientes (1967) tuvieron una puntuación mayor de 12 puntos. Las mujeres tuvieron síntomas de la enfermedad de la superficie ocular con más frecuencia que los hombres (odds ratio [OR]: 1,17; intervalo de confianza del 95\%: 1.08-1.28) y la puntuación OSDI más alta (42.12 \pm 24.03 vs. $38.01 \pm 22.81$ puntos). Los pacientes sin enfermedad fueron más jóvenes que aquellos con enfermedad grave (45.30 \pm 18.32 vs. $50.62 \pm 18.86$ ). Conclusiones: Los pacientes oftalmológicos tienen una prevalencia del $80.4 \%$ de enfermedades de la superficie ocular. El sexo femenino y la edad avanzada se asociaron más con enfermedad de la superficie ocular.

PALABRAS CLAVE: Enfermedad de la superficie ocular. Cuestionario IESO. Ojo seco. Factores de riesgo. Pacientes oftalmológicos.

\begin{abstract}
Objective: To determine the prevalence of symptoms of ocular surface disease (OSDI) surface disease and its relationship with associated risk factors in patients of ophthalmic practices using OSDI questionnaire. Method: $A$ cross-sectional survey was conducted Between September and December 2014 to assess the prevalence and risk factors for OSDI. Results: The OSDI average value was $40.46 \pm 23.62$ points, with $86.4 \%$ of patients (1967) having a OSDI score higher than 12 points. Women had OSDI symptoms more frequently than men (odds ratio: 1.17; 95\% confidence interval: 1.08-1.28) and higher OSDI score (42.12 \pm 24.03 vs. $38.01 \pm 22.81$ points). Patients without disease were younger than the patients with severe disease (45.30 \pm 18.32 vs. $50.62 \pm 18.86$ ). Conclusions: Ophthalmological patients have a prevalence of $80.4 \%$ of OSDI. Female and older age was associated with ocular surface disease.
\end{abstract}

KEY WORDS: Ocular surface disease. Ocular surface disease questionnaire. Dry eye. Risk factors. Ophthalmic patients.

\author{
Correspondencia: \\ Manuel Garza-León \\ Avda. Hidalgo, 2425 Poniente \\ Col. Obispado \\ C.P. 64060 , Monterrey, N.L., México \\ E-mail: manuel.garza@udem.edu
}

Fecha de recepción: 23-09-2016

Fecha de aceptación: 06-12-2016

DOI://dx.doi.org/10.24875/GMM.17002885
Gac Med Mex. 2017;153:769-774

Contents available at PubMed www.gacetamedicademexico.com 


\section{Introducción}

El ojo seco es una de las enfermedades más comunes y una causa frecuente de consulta oftalmológica'. Es una enfermedad multifactorial de la superficie ocular que afecta a la película lagrimal provocando su inestabilidad, dando como resultado síntomas de malestar ocular, alteraciones visuales y un potencial daño de las estructuras oculares externas ${ }^{2}$.

La prevalencia de la enfermedad ha sido ampliamente estudiada con resultados muy variables, desde el $5 \%$ hasta más del $70 \%$ en personas mayores de 50 años ${ }^{1,3-13}$; los resultados varían de acuerdo con la definición utilizada, los métodos diagnósticos y la población estudiada'.

El cuestionario del Índice de Enfermedad de la Superficie Ocular (OSDI, por sus siglas en inglés) es un método muy utilizado en estudios epidemiológicos de ojo seco y enfermedad de la superficie ocular, ya que evalúa por medio de 12 preguntas no solo la sintomatología asociada a esta enfermedad, sino también el impacto en la calidad de vida de los encuestados. Los resultados de la encuesta OSDI pueden tener valores entre 0 y 100 puntos, y según la guía publicada sobre el resultado del OSDI podemos dividir a los pacientes en aquellos que tienen una superficie ocular normal (0 a 12 puntos), enfermedad leve de la superficie ocular (13 a 22 puntos), enfermedad moderada ( 23 a 32 puntos) y grave (33 a 100 puntos) ${ }^{14}$. Para la realización de nuestro estudio utilizamos el cuestionario OSDI en una versión validada al españo ${ }^{15}$.

Hasta nuestro conocimiento, en México no existe información publicada sobre la prevalencia de síntomas de enfermedad de la superficie ocular en pacientes que acuden a consulta oftalmológica, y siendo este un problema de salud pública ${ }^{16}$ es imprescindible contar con información al respecto en nuestro país.

El objetivo es conocer la prevalencia de síntomas de enfermedad de la superficie ocular en pacientes que acuden a consulta oftalmológica por primera vez en nuestro país, así como conocer la relación entre los síntomas, la edad y el sexo de los pacientes; y finalmente comparar la prevalencia entre los pacientes que acuden a consulta institucional y privada.

\section{Método}

Se realizó entre septiembre y diciembre de 2014 en centros públicos y privados de alta especialidad en oftalmología, por los médicos que pertenecen al Grupo Mexicano de Investigación en Ciencias Visuales localizados en 19 de los 32 Estados de la República Mexicana. Se utilizó el cuestionario OSDI traducido y validado al español ${ }^{15}$ en los pacientes que acudieron a consulta oftalmológica por primera vez, para ser respondido de forma autónoma antes de ingresar al consultorio. En caso de que el paciente tuviese alguna duda, esta se resolvió de forma directa por el médico especialista.

Se incluyeron de manera consecutiva pacientes mayores de 18 años, de ambos sexos, que accedieron a participar voluntariamente en el estudio. Se excluyeron los pacientes que presentaron cualquier cuadro de infección activa de la superficie ocular, alteraciones estructurales o funcionales de los párpados, incluidas secuelas de parálisis facial, o bien alguna alteración mental que comprometiera la comprensión del cuestionario.

La captura de los datos se realizó por vía Internet mediante un sitio creado específicamente para ello. Además del cuestionario OSDI, se capturaron los siguientes datos: identificación, fecha de llenado del cuestionario, fecha de nacimiento, sexo, lugar de referencia del paciente (institucional o consultorio privado), nombre del médico y comentarios adicionales.

El análisis estadístico se realizó con el programa estadístico R (R Core Central, 2015) para analizar los resultados y con el software Stan (Equipo de Desarrollo de Stan. 2016. RStan: la interfaz $\mathrm{R}$ a Stan, Versión 2.9.0. http://mc-stan.org) para el modelo de regresión bayesiano beta.

De acuerdo con estudios previos en nuestra población (datos no publicados), nuestra hipótesis es que la prevalencia de sintomatología de enfermedad de la superficie ocular es mayor del $50 \%$.

\section{Resultados}

De 2270 participantes, 1348 (59.8\%) fueron mujeres y $922(40.2 \%)$ fueron hombres, con una edad promedio de $50 \pm 17.68$ años (rango: 18-97). Las mujeres tuvieron una edad promedio de $50.98 \pm 17.82$ años y los hombres de $48.56 \pm 17.37$ años; la diferencia en la edad no fue estadísticamente significativa $(p=0.154)$. Las características demográficas se presentan en la tabla 1. Aproximadamente el $75 \%$ de los pacientes respondieron todas las preguntas ${ }^{12}$, el $9 \%$ que respondieron 11 y el $5 \%$ respondieron 10 ; el mínimo de preguntas respondidas fue de cinco en un $1.5 \%$ de los cuestionarios. 
Tabla 1. Datos demográficos

\begin{tabular}{|c|c|c|c|c|c|}
\hline & $\begin{array}{c}\text { Pacientes } \\
n=2,270(100 \%)\end{array}$ & $\begin{array}{l}\text { Sin enfermedad } \\
n=303(13.3 \%)\end{array}$ & $\begin{array}{c}\text { Enfermedad leve } \\
n=308(13.6 \%)\end{array}$ & $\begin{array}{l}\text { Enfermedad moderada } \\
\qquad n=305(13.4 \%)\end{array}$ & $\begin{array}{c}\text { Enfermedad grave } \\
n=1,354(59.6 \%)\end{array}$ \\
\hline Edad \pm DE (IC 95\%) & $50 \pm 17.68$ & $\begin{array}{l}45.30 \pm 18.32 \\
(43.14-47.45)\end{array}$ & $\begin{array}{l}45.15 \pm 19.08 \\
(42.79-47.49)\end{array}$ & $\begin{array}{l}48.15 \pm 18.78 \\
(46.17-50.13)\end{array}$ & $\begin{array}{l}50.62 \pm 18.86 \\
(49.59-51.65)\end{array}$ \\
\hline Hombres, n (\%) & $922(40.6)$ & $146(15.8)$ & 125 (13.6) & $135(14.6)$ & $516(56)$ \\
\hline Mujeres, n (\%) & $1348(59.4)$ & 157 (11.6) & $183(13.6)$ & $170(12.6)$ & 838 (62.2) \\
\hline
\end{tabular}

DE: desviación estándar; IC 95\%: intervalo de confianza del 95\%.

El valor promedio del resultado del cuestionario OSDI fue de $40.46 \pm 23.62$ unidades. Presentaron síntomas de enfermedad superficie ocular 1967 participantes (86.4\%).

Los pacientes sin síntomas fueron más jóvenes que los demás (tabla 1), siendo la diferencia de edad estadísticamente significativa entre los pacientes sin síntomas o síntomas leves y aquellos con sintomatología grave. En relación con el sexo, las mujeres tuvieron valores del cuestionario OSDI mayores que los hombres ( $p=0.001$ ), $42.12 \pm 24.03$ vs. $38.01 \pm 22.81$ puntos, respectivamente, y además mostraron síntomas más graves que los hombres (tabla 1).

La tabla 2 muestra los efectos estimados de los factores de riesgo (a través del modelo de regresión Beta). Las mujeres son propensas a presentar valores de OSDI más altos que los hombres (OR: 1.17; intervalo de confianza del 95\% [IC 95\%]: 1.08-1.28). Los pacientes de mayor edad son propensos a presentar valores de OSDI más altos (RR: 1.32; IC 95\%: 1.211.43). Los pacientes de médicos especialistas en enfermedades inflamatorias oculares y retina tienen una tendencia a presentar menores valores OSDI que aquellos que provenían de médicos especialistas en segmento anterior (RR: 0.44, IC 95\%: 0.34-0.57; RR: 0.82, IC 95\%: 0.67-0.98; respectivamente).

La prevalencia de síntomas de enfermedad de la superficie ocular según las décadas de la edad se presenta en la tabla 3 . El resultado del cuestionario OSDI más alto fue en los pacientes de 91 a 100 años, seguido de los de 81 a 90 años y los de 71 a 80 años. Aunque en todas las décadas de la vida predominó la sintomatología grave, la mayor frecuencia de enfermedad grave se encontró a partir de la sexta década de la vida, con excepción de los 91 a 100 años, grupo en que el número de pacientes fue muy pequeño, por lo que la frecuencia de enfermedad grave no fue tan alta aun cuando el resultado del cuestionario OSDI fue el más alto de todos los grupos de edad.

Los pacientes provinieron de médicos con diferentes estudios de alta especialidad, casi la mitad (1073;
Tabla 2. Efectos estimados con riesgo relativo de los factores de riesgo

\begin{tabular}{lcc}
\hline Factor & RR & IC 95\% \\
\hline Mujer & 1.17 & $1.08-1.28$ \\
Edad & 1.32 & $1.21-1.43$ \\
\multicolumn{3}{l}{ Especialidad del doctor (vs. segmento anterior) } \\
Enfermedades inflamatorias oculares & 0.44 & $0.34-0.57$ \\
Retina & 0.82 & $0.67-0.98$ \\
Córnea & 1.01 & $0.90-1.14$ \\
Glaucoma & 1.05 & $0.90-1.23$ \\
\hline
\end{tabular}

IC 95\%: intervalo de confianza del 95\%; RR: Riesgo relativo.

47.3\%) de doctores especialistas en córnea, seguidos de $493(21.7 \%)$ de doctores de segmento anterior, $326(14.4 \%)$ de doctores de glaucoma, 290 (12.8) de doctores de retina y $88(3.9 \%)$ de especialistas en enfermedades inflamatorias oculares. Los valores de OSDI por especialidad se muestran en la tabla 4.

Los pacientes evaluados provenían tanto de instituciones públicas como de consultorios privados (el $81.4 \%$ de los pacientes provenían de consultorios privados). Al evaluar si la fuente del paciente tenía un efecto en la prevalencia de enfermedad de la superficie ocular, a través de la prueba de ji al cuadrado se encontró que no hubo una asociación entre la fuente del paciente y la frecuencia de enfermedad de la superficie ocular ( $p=0.493)$.

\section{Discusión}

La prevalencia de ojo seco en la población general presenta una gran variación, que va desde un 7.8 hasta un 57.5\% $\%^{17-22}$ dependiendo de la definición de ojo seco y de los estudios diagnósticos realizados; sin embargo, cuando se toma en cuenta únicamente la sintomatología, la prevalencia de síntomas de ojo seco puede ser tan alta como el $93.2 \%$ reportado por Bukhari, et al. ${ }^{3}$, quienes estudiaron a 251 acompañantes de pacientes que acudieron a consulta oftalmológica en Jeddah, Arabia Saudita. En nuestro estudio se calculó la prevalencia de síntomas de enfermedad de la superficie 
Tabla 3. Resultados del cuestionario OSDI por edad en décadas

\begin{tabular}{lcccccc}
\hline Rango edad, años & $\mathbf{n}$ & Valor OSDI & $\begin{array}{c}\text { Sin enfermedad, } \\
\mathbf{n}(\%)\end{array}$ & $\begin{array}{c}\text { Enfermedad } \\
\text { leve, } \mathbf{n}(\%)\end{array}$ & $\begin{array}{c}\text { Enfermedad } \\
\text { moderada, } \mathbf{n}(\%)\end{array}$ & $\begin{array}{c}\text { Enfermedad } \\
\mathbf{g r a v e , ~} \mathbf{n}(\%)\end{array}$ \\
\hline 18 a 30 & 370 & $35.93 \pm 2.69$ & $61(16.5)$ & $64(17.3)$ & $42(11.4)$ & $203(54.9)$ \\
31 a 40 & 380 & $35.74 \pm 1.26$ & $65(17.1)$ & $67(17.6)$ & $57(15)$ & $191(50.3)$ \\
41 a 50 & 399 & $40.10 \pm 1.23$ & $54(13.5)$ & $55(13.8)$ & $49(12.3)$ & $241(60.4)$ \\
51 a 60 & 449 & $44.29 \pm 1.15$ & $48(10.7)$ & $51(11.4)$ & $62(13.8)$ & $288(64.1)$ \\
61 а 70 & 336 & $41.67 \pm 1.33$ & $44(13.1)$ & $37(11 \%)$ & $47(14)$ & $208(61.9)$ \\
71 a 80 & 243 & $44.40 \pm 1.55$ & $25(10.3)$ & $25(10.3$ & $38(15.6)$ & $155(63.8)$ \\
81 a 90 & 91 & $48.40 \pm 2.50$ & $6(6.6)$ & $8(8.8)$ & $10(11)$ & $67(73.6)$ \\
91 a 100 & 2 & $54.36 \pm 16.5$ & $0(0)$ & $1(50)$ & $0(0)$ & $1(50)$ \\
\hline
\end{tabular}

Tabla 4. Valor de la puntuación OSDI por especialidad del médico de referencia

\begin{tabular}{lccc}
\hline Especialidad & $\mathbf{n}$ & Promedio \pm DE & IC 95\% \\
\hline Córnea & 1,073 & $41.79 \pm 23.33$ & $40.39-43.19$ \\
Segmento anterior & 493 & $41.31 \pm 23.45$ & $39.23-43.38$ \\
Glaucoma & 326 & $42.06 \pm 24.64$ & $39.38-44.75$ \\
Retina & 290 & $39.30 \pm 22.82$ & $36.66-41.94$ \\
Enfermedades inflamatorias oculares & 88 & $25.45 \pm 20.25$ & $21.16-29.74$ \\
\hline
\end{tabular}

DE: desviación estándar; IC 95\%: intervalo de confianza del 95\%.

ocular en pacientes oftalmológicos; existen pocos estudios que evalúen pacientes que acuden a consulta oftalmológica $^{23,24}$ o a consulta optométrica ${ }^{25,26}$. En nuestro estudio se encontró una prevalencia de síntomas del $86.4 \%$, la cual es mucho mayor que en otros estudios similares, como el de Hikichi, et al. ${ }^{23}$, que la reportan en un $17 \%$ de los adultos que acudieron a consulta oftalmológica en ocho centros de Japón; sin embargo, en su estudio se excluyó a todos los pacientes con sintomatología de ojo seco (89 de 2127), el cuestionario utilizado fue diferente y además el diagnóstico de ojo seco se realizó con estudios clínicos, y de acuerdo a lo reportado por numerosos autores no existe una correlación importante entre los síntomas y signos de ojo seco ${ }^{27-30}$. Resultados similares encuentran Sahai y Malik ${ }^{24}$ al evaluar 500 pacientes que acudieron a consulta oftalmológica en Jaipur, India, utilizando el mismo cuestionario que Hikichi, et al. ${ }^{23}$ junto con estudios clínicos como tinciones vitales con verde de lisamina y fluoresceína, prueba de Schirmer y tiempo de rotura lagrimal, reportando una prevalencia del 18.4\%; sin embargo, el 55.6\% refirió visión borrosa, que es uno de los síntomas evaluados en el cuestionario OSDI, lo que confirma la dificultad para comparar estos estudios con el realizado por nosotros. Albietz ${ }^{25}$ evaluó a 1584 pacientes que acudieron por primera vez a una consulta optométrica en Queensland, Australia, y utilizando el cuestionario de McMonnies, además de tinciones vitales con fluoresceína y rosa de bengala, y medición del tiempo de rotura lagrimal, encontró una prevalencia de ojo seco del $10.8 \%$, valor menor que el reportado por Doughty, et al. ${ }^{26}$, quienes evaluaron la prevalencia de síntomas de ojo seco utilizando un cuestionario de 13 puntos (The Canada Dry Eye Epidemiology Study [CANDEES]) en nueve provincias de Canadá y el $28.7 \%$ de los participantes respondieron positivamente a la pregunta de si tenían síntomas de ojo seco.

Las puntuaciones del cuestionario OSDI fueron mayores en las mujeres que en los hombre de forma estadísticamente significativa (38.01 \pm 22.81 vs. $42.12 \pm 24.03$, respectivamente), debido tanto a que la prevalencia de síntomas de enfermedad de la superficie ocular fue mayor en las mujeres que en los hombres (88 vs. 84.9\%) como a que las mujeres presentaron mayor frecuencia de sintomatología grave que los hombres (61.5 vs. 55.3\%); esto es similar a lo hallado en la mayoría de los estudios sobre ojo seco, en los que se observa que las mujeres se afectan más frecuentemente que los hombres ${ }^{1,4,28,31-33}$. En nuestro 
estudio, además de la influencia de los cambios relacionados con el sexo, también podría estar influyendo que las mujeres tuvieron una mayor edad que los hombres, y tanto en nuestro estudio como en otros ${ }^{4,25,26,31,34}$ la edad tiene una relación directamente proporcional con la enfermedad de la superficie ocular.

Los pacientes que provenían de médicos con alta especialidad en retina y enfermedades inflamatorias oculares tuvieron los valores OSDI más bajos; esto pudiera deberse a que, aunque algunos autores han encontrado una mayor prevalencia de ojo seco y disfunción de glándulas de Meibomio en pacientes diabéticos ${ }^{35-37}$, quienes son la población con enfermedad retiniana más frecuente en México, Fuerst, et al. ${ }^{37}$ encontraron que cuanto mayor sea el tiempo de evolución con diabetes, menor sintomatología presentan, además de que el número de pacientes fue el segundo más bajo, solo por encima de los pacientes de doctores especialistas en enfermedades inflamatorias oculares. En relación con los pacientes de doctores especialistas en enfermedades inflamatorias oculares, aunque muchas de las enfermedades asociadas a estas presentan una alta prevalencia de ojo seco ${ }^{38-41}$, el uso de esteroides produce una disminución importante de los síntomas asociados a enfermedad de la superficie ocular ${ }^{42-46}$.

En conclusión, nuestro estudio encontró una muy alta prevalencia de síntomas de enfermedad de la superficie ocular en los pacientes que acuden a consulta oftalmológica en México, siendo el sexo femenino y el aumento de la edad los factores de riesgo más importantes.

Miembros del Grupo Mexicano de Investigación en Ciencias Visuales que participaron en este estudio: Adaí Pérez Montecinos, Adolfo Peña Aceves, Alejandro del Castillo Ruiz, Alejandro Franco, Arturo Jaime Gallegos Valencia, Arturo Luis Villarreal Muraira, Carlos Carral, Cynthia Espinosa, Diana Báez, Everardo Hernández Quintela, Fabiola Barrera Pelayo, Felipe Hernández, Fernando Aguilera, Fernando Rodríguez Sixtos, Francisco Segura, Gerardo García, Gonzalo Padilla, Héctor Cámara, Jorge Pacheco Patrón, Jorge Rivera Sempertegui, Juan C. Ochoa Tabares, Karen Hernández, Karina Soto Ortiz, Karla Chávez, Manuel Garza León, Nicolás León Pérez, Óscar del Razo, Paola Covarrubias, Paola de la Parra Colín, Ricardo Leal Rodríguez, Roberto González, Roberto Leal, Silvia Elena Vílchez Riestra, Susana Martínez Jardón y Valeria Sánchez Huerta.

\section{Bibliografía}

1. The epidemiology of dry eye disease: report of the Epidemiology Subcommittee of the International Dry Eye WorkShop (2007). Ocul Surf. 2007;5:93-107.
2. The definition and classification of dry eye disease: report of the Definition and Classification Subcommittee of the International Dry Eye WorkShop (2007). Ocul Surf. 2007;5:75-92.

3. Bukhari A, Ajlan R, Alsaggaf $H$. Prevalence of dry eye in the normal population in Jeddah, Saudi Arabia. Orbit (Amsterdam, Netherlands). 2009;28:392-7.

4. Ahn JM, Lee SH, Rim TH, et al. Prevalence of and risk factors associated with dry eye: the Korea National Health and Nutrition Examination Survey 2010-2011. Am J Ophthalmol. 2014;158:1205-14.e7.

5. Gupta RC, Ranjan R, Kushwaha RN, et al. A questionnaire-based survey of dry eye disease among leather tannery workers in Kanpur, India: a case-control study. Cutan Ocul Toxicol. 2014;33:265-9.

6. Li J, Zheng K, Deng Z, et al. Prevalence and risk factors of dry eye disease among a hospital-based population in southeast China. Eye Contact Lens. 2015;41:44-50.

7. Malet F, Le Goff M, Colin J, et al. Dry eye disease in French elderly subjects: the Alienor Study. Acta Ophthalmol. 2014;92:e429-36.

8. Paulsen AJ, Cruickshanks KJ, Fischer ME, et al. Dry eye in the beaver dam offspring study: prevalence, risk factors, and health-related quality of life. Am J Ophthalmol. 2014;157:799-806.

9. Tan LL, Morgan P, Cai ZQ, et al. Prevalence of and risk factors for symptomatic dry eye disease in Singapore. Clin Exp Optom. 2015; 98:45-53.

10. Uchino M, Uchino $Y$, Dogru $M$, et al. Dry eye disease and work productivity loss in visual display users: the Osaka study. Am J Ophthalmol. 2014;157:294-300.

11. Uchino M, Yokoi N, Uchino Y, et al. Prevalence of dry eye disease and its risk factors in visual display terminal users: the Osaka study. Am J Ophthalmol. 2013;156:759-66.

12. Vehof J, Kozareva D, Hysi PG, et al. Prevalence and risk factors of dry eye disease in a British female cohort. Br J Ophthalmol. 2014;98:1712-7.

13. Uchino M, Dogru M, Yagi $Y$, et al. The features of dry eye disease in a Japanese elderly population. Optom Vis Sci. 2006;83:797-802.

14. Miller KL, Walt JG, Mink DR, et al. Minimal clinically important difference for the ocular surface disease index. Arch Ophthalmol. 2010;128:94-101.

15. Beltrán F, Ramos Betancourt N, Martínez J, et al. Transcultural validation of Ocular Surface Disease Index (OSDI) questionnaire for Mexican population. Invest Ophthalmol Vis Sci. 2013;54:6050.

16. Uchino M, Schaumberg DA. Dry eye disease: impact on quality of life and vision. Current Ophthalmology Reports. 2013;1:51-7.

17. Chia EM, Mitchell $P$, Rochtchina E, et al. Prevalence and associations of dry eye syndrome in an older population: the Blue Mountains Eye Study. Clin Exp Ophthalmol. 2003;31:229-32.

18. Farrell J, Grierson DJ, Patel S, et al. A classification for dry eyes following comparison of tear thinning time with Schirmer tear test. Acta Ophthalmol (Copenh). 1992;70:357-60.

19. McCarty CA, Bansal AK, Livingston PM, et al. The epidemiology of dry eye in Melbourne, Australia. Ophthalmology. 1998;105:1114-9.

20. Toda I, Fujishima H, Tsubota K. Ocular fatigue is the major symptom of dry eye. Acta Ophthalmol (Copenh). 1993;71:347-52.

21. Tong L, Saw SM, Lamoureux EL, et al. A questionnaire-based assessment of symptoms associated with tear film dysfunction and lid margin disease in an Asian population. Ophthalmic Epidemiol. 2009;16:31-7.

22. Versura $P$, Cellini $M$, Torreggiani $A$, et al. Dryness symptoms, diagnostic protocol and therapeutic management: a report on 1,200 patients. Ophthalmic Res. 2001;33:221-7.

23. Hikichi T, Yoshida A, Fukui Y, et al. Prevalence of dry eye in Japanese eye centers. Graefes Arch Clin Exp Ophthalmol. 1995;233:555-8.

24. Sahai A, Malik P. Dry eye: prevalence and attributable risk factors in a hospital-based population. Indian J Ophthalmol. 2005;53:87-91.

25. Albietz JM. Prevalence of dry eye subtypes in clinical optometry practice. Optom Vis Sci. 2000;77:357-63.

26. Doughty MJ, Fonn D, Richter D, et al. A patient questionnaire approach to estimating the prevalence of dry eye symptoms in patients presenting to optometric practices across Canada. Optom Vis Sci. 1997; 74:624-31.

27. Begley CG, Chalmers RL, Abetz L, et al. The relationship between habitual patient-reported symptoms and clinical signs among patients with dry eye of varying severity. Invest Ophthalm Vis Sci. 2003;44:4753-61.

28. Lin PY, Cheng CY, Hsu WM, et al. Association between symptoms and signs of dry eye among an elderly Chinese population in Taiwan: the Shihpai Eye Study. Invest Ophthalm Vis Sci. 2005;46:1593-8.

29. Nichols KK, Nichols JJ, Mitchell GL. The lack of association between signs and symptoms in patients with dry eye disease. Cornea. 2004;23:762-70.

30. Schein OD, Tielsch JM, Munoz B, et al. Relation between signs and symptoms of dry eye in the elderly. A population-based perspective. Ophthalmology. 1997;104:1395-401.

31. Galor A, Feuer W, Lee DJ, et al. Prevalence and risk factors of dry eye syndrome in a United States veterans affairs population. Am J Ophthalmol. 2011;152:377-84.e2.

32. Schaumberg DA, Buring JE, Sullivan DA, et al. Hormone replacement therapy and dry eye syndrome. JAMA. 2001;286:2114-9. 
33. Schaumberg DA, Sullivan DA, Buring JE, et al. Prevalence of dry eye syndrome among US women. Am J Ophthalmol. 2003;136:318-26.

34. Guo B, Lu P, Chen X, et al. Prevalence of dry eye disease in Mongolians at high altitude in China: the Henan eye study. Ophthalm Epidemiol. 2010;17:234-41.

35. Najafi L, Malek M, Valojerdi AE, et al. Dry eye and its correlation to diabetes microvascular complications in people with type 2 diabetes mellitus. J Diabetes Complications. 2013;27:459-62.

36. Shamsheer RP, Arunachalam C. A clinical study of Meibomian gland dysfunction in patients with diabetes. Middle East Afr J Ophthalmol. 2015;22:462-6.

37. Fuerst N, Langelier N, Massaro-Giordano M, et al. Tear osmolarity and dry eye symptoms in diabetics. Clin Ophthalmol (Auckland, NZ) 2014:8:507-15.

38. Berchicci L, luliano L, Miserocchi E, et al. Tear osmolarity in ocular graftversus-host disease. Cornea. 2014;33:1252-6.

39. Kotaniemi KM, Salomaa PM, Sihto-Kauppi K, et al. An evaluation of dry eye symptoms and signs in a cohort of children with juvenile idiopathic arthritis. Clin Ophthalmol (Auckland, NZ). 2009;3:271-5.
40. Miserocchi E, luliano L, Berchicci L, et al. Tear film osmolarity in ocular mucous membrane pemphigoid. Cornea. 2014;33:668-72.

41. Zeng J, Chen B. Severe primary ocular surface involvement in Behçet disease. Optom Vis Sci. 2014;91:e301-4.

42. Moore QL, De Paiva CS, Pflugfelder SC. Effects of dry eye therapies on environmentally induced ocular surface disease. Am J Ophthalmol. 2015;160:135-42.e1.

43. Pinto-Fraga J, López-Miguel A, González-García MJ, et al. Topical fluorometholone protects the ocular surface of dry eye patients from desiccating stress: a randomized controlled clinical trial. Ophthalmology. 2016;123:141-53.

44. Villani E, Garoli E, Termine V, et al. Corneal confocal microscopy in dry eye treated with corticosteroids. Optom Vis Sci. 2015:92:e290-5.

45. Boynton GE, Raoof D, Niziol LM, et al. Prospective randomized trial comparing efficacy of topical loteprednol etabonate $0.5 \%$ versus cyclosporine-A $0.05 \%$ for treatment of dry eye syndrome following hematopoietic stem cell transplantation. Cornea. 2015;34:725-32.

46. Lee JH, Min K, Kim SK, et al. Inflammatory cytokine and osmolarity changes in the tears of dry eye patients treated with topical $1 \%$ methylprednisolone. Yonsei Med J. 2014:55:203-8. 